Check for updates

Cite this: RSC Adv., 2018, 8, 23404

\title{
In vivo detection of salicylic acid in sunflower seedlings under salt stress $\uparrow$
}

\begin{abstract}
Ye Hu, ${ }^{\text {ab }}$ Jing Zhao, ${ }^{\text {ac }}$ Haiyang $\mathrm{Li}^{,}{ }^{\mathrm{a}}$ Xiaodong Wang, ${ }^{\mathrm{ab}}$ Peichen Hou, ${ }^{\text {ab }}$ Cheng Wang, ${ }^{\text {ab }}$ Aixue Li ${ }^{*}{ }^{* a b}$ and Liping Chen ${ }^{\star a b}$

Salicylic acid (SA) is an important phytohormone. It plays an essential role in regulating many physiological processes of plants. Most of the conventional methods for SA detection are based on in vitro processes. More attention should be paid to develop in vivo methods for SA detection. In this work, Pt nanoflowers and GO were simultaneously electrodeposited and reduced on a Pt wire microelectrode in one step. The Pt nanoflowers/ERGO modified Pt microsensor demonstrated high sensitivity and selectivity for SA. SA could be detected from $100 \mathrm{pM}$ to $1 \mu \mathrm{M}$ with a detection limit of $48.11 \mathrm{pM}$. Then this microsensor was used to detect SA in the stem of sunflower seedlings under different salt stresses in vivo. The result showed that with the increasing concentration of salt, SA levels decreased. Our result was also confirmed by UPLC-MS and gene expression analysis. To the best of our knowledge, this is the first report of in vivo detection of SA in plants using the Pt nanoflowers/ERGO modified Pt microelectrode. It is foreseeable that our strategy could pave the way for the in vivo detection of phytohormones in plants.
\end{abstract}

Received 23rd April 2018 Accepted 21st June 2018

DOI: $10.1039 / c 8 r a 03475 c$

rsc.li/rsc-advances always required, and some biological information may be lost during this process. In addition, the collections of plant samples always result in great damage and even death, while many plant materials are very precious and rare. More importantly, an in vivo detection of SA would provide more instant and accurate information about the response mechanism of plants under different environmental conditions. Therefore, it is very important to develop in vivo method for the detection of SA.

To date, there are only a few reports for the in vivo detection of SA, such as colorimetry, ${ }^{8}$ bioluminescence, ${ }^{9}$ reverse iontophoresis $^{\mathbf{1 0}}$ and electrochemical sensors. ${ }^{\mathbf{1 1}}$ Colorimetry and bioluminescence methods were mainly used to map the spatial distribution of SA in plant tissues. Reverse iontophoresis has been used to assay the level of SA in basil leaves in situ, but it needed an 8 hours extraction period for collecting the sap of the plants, which couldn't reflect the instant information of SA under changed environment. Sun et al. ${ }^{11}$ detected the change of SA in tomato leaves in vivo using a paper-based electrochemical sensor, which indicated that electrochemical sensor could be used for in situ and in vivo detection of SA in plants. However, it seems that this sensor is more suitable for the leaves, while not suitable for the stems or roots of the plants.

In recent years, microelectrode has attracted much interest due to its unique characters, such as smaller size, rapid response time, increased signal-to-noise ratio, and less requirement for support electrolyte, etc. ${ }^{12,13}$ Additionally, the small size of microelectrode makes it ideal for in vivo application in plants, especially for the stems or roots of plants. For example, Pt microelectrode has been successfully used to monitor the $\mathrm{H}_{2} \mathrm{O}_{2},{ }^{14-17} \mathrm{NO}$ and $\mathrm{pH}^{15}$ in plants in vivo. However,
${ }^{a}$ Beijing Research Center of Intelligent Equipment for Agriculture, Beijing Academy of nercita.org.cn

${ }^{b}$ Beijing Research Center for Information Technology in Agriculture, Beijing Academy of Agriculture and Forestry Sciences, Beijing 100097, P. R. China. E-mail: chenlp@ nercita.org.cn

${ }^{c}$ Department of Environmental Science and Engineering, Zhejiang Ocean University, Zhoushan, Zhejiang 316022, P. R. China

$\dagger$ Electronic supplementary information (ESI) available. See DOI: $10.1039 / \mathrm{c} 8 \mathrm{ra} 03475 \mathrm{c}$ 
up to date, there are few reports about the application of microelectrode in detecting of phytohormone in plants in vivo.

As the environment for in vivo detection is very complex, complicated and complex method for fabrication of the microsensor is not suitable. It has been reported that electrochemically reduced graphene oxide (ERGO)-metal nanocomposite films can be obtained by direct electrodeposition method. ${ }^{18,19}$ This method is green and fast, and the prepared nanocomposite films exhibit excellent catalytic activity. In this work, Pt nanoflowers (PtNF) and ERGO were successfully fabricated on a Pt wire microelectrode by one step electrodeposition method. Then this PtNF/ERGO modified Pt microelectrode was used to detect SA in the stem of sunflower seedlings under salt stress in vivo. To the best of our knowledge, this is the first report for in vivo detection of SA in plants using the PtNF/ERGO modified Pt microelectrode. It may open the opportunity for application of microelectrode in detecting of phytohormone in vivo in plants. Our result was also confirmed by UPLC-MS and gene expression analysis.

\section{Materials and methods}

\subsection{Chemicals and materials}

Hexachloroplatinic acid hexahydrate $\left(\mathrm{H}_{2} \mathrm{PtCl}_{6} \cdot 6 \mathrm{H}_{2} \mathrm{O}\right)$, SA, methyl jasmonate, malic acid, succinic acid, citric acid, indole acetic acid and abscisic acid were purchased from Sigma-Aldrich Co. LLC. The monolayer graphene oxide (GO) (diameter: $500 \mathrm{~nm}$ to $5 \mu \mathrm{m}$ ) was obtained from Nanjing XFNANO Materials Tech Co. Ltd. All other chemicals used were analytically grade and purchased from Beijing Chemical Works. All aqueous solutions were prepared with ultrapure water. The SA stock solution ( $0.01 \mathrm{M})$ was dissolved by $0.01 \mathrm{M}$ PBS $(\mathrm{pH}=7.0)$ with $0.2 \%$ ethanol. It was stored under $4{ }^{\circ} \mathrm{C}$ and diluted freshly by PBS before use.

\subsection{Preparation of the Pt nanoflowers/ERGO modified Pt (PtNF/ERGO/Pt) microelectrodes}

Pt wire microelectrode $(0.5 \mathrm{~mm}$ in diameter) was purchased from Tianjin Ida Heng Sheng Technology Development Co., Ltd (Tianjin, China). The microelectrode was immersed in concentrated nitric acid solution for $10 \mathrm{~min}$ to remove the impurities. Then, it was polished in turn with 0.3 and $0.05 \mu \mathrm{m}$ alumina powder, and ultrasonic cleaning with deionized water and ethanol. After drying by $\mathrm{N}_{2}$, the Pt microelectrode was voltammetry cycled in $0.1 \mathrm{M}$ sulfuric acid with the potential from -1.5 to $1.5 \mathrm{~V}$ for 30 cycles.

The cleaned Pt wire microelectrode was potential scanned in GO aqueous suspension (0.1 $\mathrm{M}$ carbonate buffer at $\mathrm{pH} 9.0)$ containing $\mathrm{H}_{2} \mathrm{PtCl}_{6}$ under the potential window from 0 to $-1.5 \mathrm{~V}$ at a scan rate of $25 \mathrm{mV} \mathrm{s}^{-1}$. The resulted PtNF/ERGO/Pt wire electrode was rinsed by deionized water and dried by nitrogen. Then it was wrapped with insulating tape, leaving $2 \mathrm{~mm}$ in length for sensing.

The ERGO/Pt and PtNF/Pt electrode was also prepared in $0.1 \mathrm{M}$ carbonate buffer contain optimal concentrations of GO or $\mathrm{H}_{2} \mathrm{PtCl}_{6}$ for control.

\subsection{Characterization}

The surface morphology of the different modified Pt wire electrodes were characterized by scanning electron microscopic (SEM,
JEOL JSM-6700F). The X-ray diffraction (XRD) analysis of the different modified Pt electrode was carried out on a SmartLab XRD system using $\mathrm{Cu} \mathrm{K} \alpha$ as the radiation source at an operating voltage of $40 \mathrm{kV}$. Pt sheet was used as the substrate to simulate the Pt wire.

\subsection{Preparation of plant samples and homogenate}

A homogenous seeds of sunflower (LD5009) were obtained from Beijing Kafry Technology Co., LTD. After seed germination, the seedlings with consistent growth were selected and transplanted into half-strength Hoagland solution. After 15 days hydroponic culture in an illuminating incubator $(10 \mathrm{~h} / 14 \mathrm{~h}$ of light/dark period, $25 \pm 2{ }^{\circ} \mathrm{C}$ and $60 \%$ humidity), the sunflowers were randomized into three groups (each group contained 20 plant samples) and treated by different salt stress for $10 \mathrm{~h}$ through introducing different levels of $\mathrm{NaCl}(0,50$ and $100 \mathrm{mM})$ into the Hoagland solution.

Homogenates of stems of sunflower seedling was prepared by homogenized the plant materials thoroughly with $0.5 \mathrm{~g}$ of crystalline silica. The homogenates were centrifuged at $10000 \mathrm{rpm}\left(10 \mathrm{~min}, 4{ }^{\circ} \mathrm{C}\right)$, and the supernatant solutions were collected and transferred to new tubes. The homogenates were prepared freshly before each measurement.

\subsection{Electrochemical measurements}

All the electrochemical experiments were performed on a $\mathrm{CHI}$ 760e electrochemical working station. A two-electrode system was employed for the measurements. The modified Pt microelectrode was used as working electrode. $\mathrm{A} \mathrm{Ag/AgCl}$ wire was used as reference/counter electrode, which was prepared by chloridizing $\mathrm{Ag}$ wire at $0.6 \mathrm{~V}$ in $0.1 \mathrm{M}$ hydrochloride acid for about $30 \mathrm{~min}$. Cyclic voltammetry (CV) was conducted from $0.6 \mathrm{~V}$ to $1.4 \mathrm{~V}$ at $50 \mathrm{mV} \mathrm{s}^{-1}$. Differential pulse voltammetry (DPV) was conducted from $0.6 \mathrm{~V}$ to $1.4 \mathrm{~V}$, and the parameters were: $0.02 \mathrm{~V}$ of increasing potential, $0.02 \mathrm{~s}$ of pulse width, $0.05 \mathrm{~V}$ of amplitude, $1 \mathrm{~s}$ of pulse period, and $0.02 \mathrm{~s}$ of sampling width.

For in vivo detection of SA in the stem of sunflower seedlings, the stem of the seedlings were punched with a puncture needle to obtain two holes. The working electrode and $\mathrm{Ag} / \mathrm{AgCl}$ electrode were placed into the holes respectively. For different plant, the electrode was placed approximately the same site as possible.

\subsection{Ultra-performance liquid chromatography-mass spectrometric (UPLC-MS) measurements}

For the UPLC-MS (Water Acquity I-Class, Waters Corporation, MA, US; Thermo Q-Exactive, Thermo Scientific, MA, US) measurements, the stem samples of the seedlings were collected and ground in liquid nitrogen immediately after 10 hours salt treatments. $1 \mathrm{~g}$ of ground sample was added $2 \mathrm{ml} 4{ }^{\circ} \mathrm{C}$ precooled methanol $(80 \%)$ and extracted overnight at $4{ }^{\circ} \mathrm{C}$. The extract was mixed with $1 \mathrm{ml}$ of $\mathrm{CHCl}_{3}$. Then the solution was vibrated at $900 \mathrm{rpm}$ for $4 \mathrm{~min}$ at $4{ }^{\circ} \mathrm{C}$. After centrifuging at $14000 \mathrm{rpm}$ for $5 \mathrm{~min}$ at $4{ }^{\circ} \mathrm{C}$, the supernatant was collected and dried by nitrogen at room temperature. The prepared sample was dissolved in $1 \mathrm{~mL}$ of methanol and filtrated by a $0.22 \mu \mathrm{m}$ microporous membrane before UPLC-MS measurements. 


\subsection{Gene expression analysis}

To confirm the result of in vivo detection of SA, semi quantitative RT-PCR analysis was performed for SA-related gene def and HaAC1. Total RNA was extracted from stem samples of sunflower seedlings ( $0.1 \mathrm{~g}$ fresh weight) with OminiPlant RNA Kit (DNase I) (ComWin Biotech, Beijing, China) according to the protocols. BioRT cDNA First Strand Synthesis Kit (Bioer, Hangzhou, China) was used to synthesis the first-strand cDNAs from $2 \mu \mathrm{g}$ total RNA. RT-PCR was performed with $1 \mu \mathrm{L}$ generated cDNA. PCR amplifications conditions: initial denaturation, $95{ }^{\circ} \mathrm{C}$ for $5 \mathrm{~min}$; 30 cycles of amplification, $94{ }^{\circ} \mathrm{C}(30 \mathrm{~s}), 58{ }^{\circ} \mathrm{C}(40$ $\mathrm{s}$ ), and $72{ }^{\circ} \mathrm{C}(40 \mathrm{~s})$; final extension, $72{ }^{\circ} \mathrm{C}$ for $7 \mathrm{~min} .6 \mu \mathrm{L}$ of the PCR product was separated on agarose gel $(1.5 \%)$.

The primers used for def were: F: $5^{\prime}$-GTGAGAAGG CAAGCCAGACA-3' and R: 5'-TCAAGGTTTGGCTGTCGCCT-3' (AF364865, referred to $\mathrm{Hu}$ et al., 2003). ${ }^{20}$ The primers used for HaAC1 were: F: 5'-CACCACCAGGGAAATGATG-3' and R: 5'TGCGGACAACTGAGAACAACA-3' (AF030301). G-Marker (EU112625) (F: 5'-GCTGTGGCCCTTGTGTTG-3' and R: $5^{\prime}$ CGCAGACCGATAGGATACCATT-30) was used as control for the comparison of amounts of cDNA in each reaction.

\section{Result and discussion}

\subsection{Characterization of the PtNF/ERGO/Pt microelectrodes}

The surface morphology of electrodes was characterized by SEM. As shown in Fig. 1A, the surface of the bare Pt microelectrode is smooth and clean. When the ERGO was formed on the Pt microelectrode (Fig. 1B), the typical crumpled and wrinkled structure of ERGO can be observed. When the PtNF was formed on the Pt microelectrode (Fig. 1C), a densely and uniformly distributed flower-like Pt nanostructure with the diameter of 200-500 nm can be observed. When the Pt and GO was codeposition and co-reduction on the Pt microelectrode, flowerlike Pt nanostructure are dispersed in the lamellar ERGO, constructing a porous network structure (Fig. 1D). The diameters of the PtNF range from 50-100 $\mathrm{nm}$. It has been reported that GO or RGO can be used to disperse and prevent the growth of the nanostructures, because they could be regarded as unconventional polymeric surfactant, ${ }^{21-23}$ so smaller size and better dispersion were observed for the PtNF with the attendance of ERGO. And ERGO can also provide anchoring site for metal nanostructures or its precursors. ${ }^{\mathbf{2 4 , 2 5}}$ Moreover, the irregularly shaped PtNF and untidy ERGO can enhance the surface area and catalytic activity of the Pt microelectrode greatly. ${ }^{2,26}$

The XRD pattern of (a) bare Pt, (b) EGRO/Pt, (c) Pt NF/Pt and (d) Pt NF/EGRO/Pt was shown in Fig. 1E. For the bare Pt (a), the peaks at $2 \theta$ of $39.8^{\circ}, 46.3^{\circ}$, and $67.8^{\circ}$ are assigned to the (111), (200), and (220) planes of the Pt particles. The peak at $2 \theta$ of $67.8^{\circ}$ is very strong, which might be ascribed to the preferred orientation of block as we used the Pt sheet to simulate the Pt wire. For the EGRO/Pt (b), a weak peak originating from graphene sheets (002) is also appeared at $2 \theta$ of about $25^{\circ}$, which confirms the reduction of GO into ERGO. ${ }^{27}$ For the Pt NF/Pt (c), the intensity of peaks of $39.8^{\circ}, 46.3^{\circ}$, and $67.8^{\circ}$ are changed compare to the bare Pt sheet (a), indicating the formation of Pt crystals. ${ }^{28}$ As expected, a peak at $2 \theta$ of $25^{\circ}$ also appears for the Pt NF/EGRO/Pt.

\subsection{Feasibility of the PtNF/ERGO/Pt microelectrode for detecting SA}

The feasibility of the modified PtNF/ERGO/Pt microelectrode for detecting SA was tested by cyclic voltammetry (CV) and

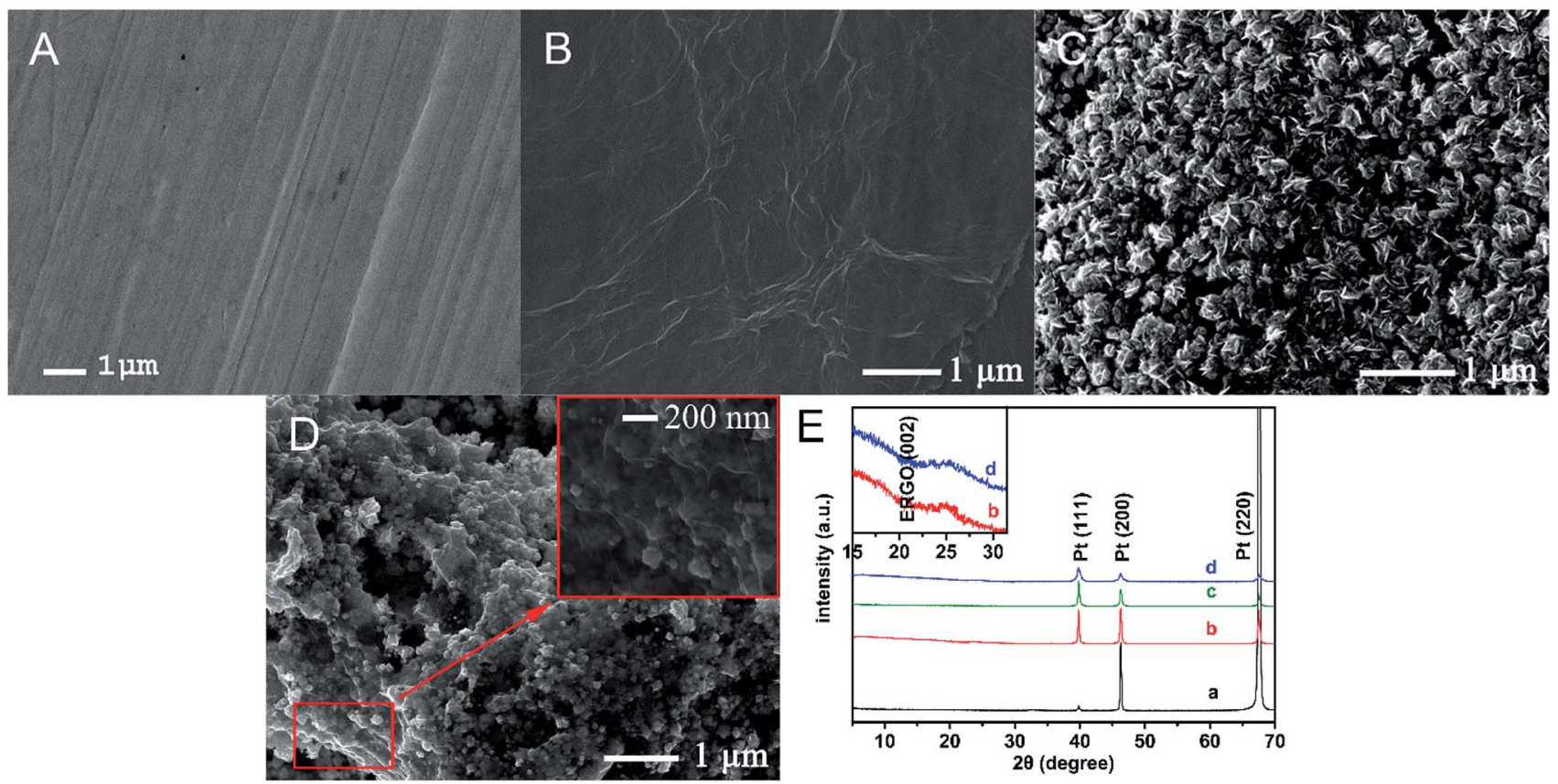

Fig. 1 SEM images of the bare Pt electrode (A), ERGO/Pt electrode (B), PtNF/Pt electrode (C), and the PtNF/ERGO/Pt electrode (D), the insert is a partial enlargement of the PtNF/ERGO/Pt electrode. (E) XRD patterns of (a) bare Pt sheet, (b) ERGO/Pt, (c) PtNF/Pt, (d) PtNF/ERGO/Pt. 

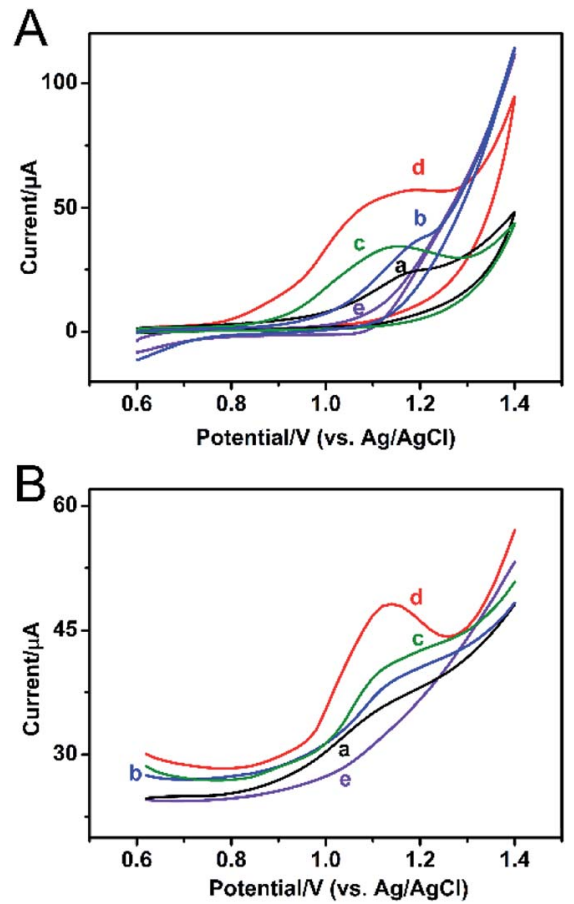

Fig. 2 (A) CV and (B) DPV curves of the bare Pt electrode (a), ERGO/Pt electrode (b), PtNF/Pt electrode(c), PtNF/ERGO/Pt electrode (d) in the presence of $500 \mu \mathrm{MSA}$, and the PtNF/ERGO/Pt electrode (e) in the PBS without SA.

differential pulse voltammetry (DPV) methods. For CV curve of $500 \mu \mathrm{M}$ SA (Fig. 2A), a single oxidation peak appeared around $1.15 \mathrm{~V}$ by the bare Pt microelectrode in the PBS buffer (curve a), which is probably attributed to the electrochemically active phenol group of SA. ${ }^{11}$ The oxidation peak was increased when the $\mathrm{ERGO} / \mathrm{Pt}$ microelectrode (curve b) and the PtNF/Pt microelectrode (curve c) were used, indicating the PtNF and ERGO can respectively enhanced the catalysis effect of SA. The oxidation peak of SA was significantly increased when the PtNF/ERGO/Pt microelectrode was used (curve d), indicating that the integration of high density Pt NF with the layered ERGO can induce more active sites and construct an electrical network, which greatly increased the catalytic capacity to SA. No oxidation peak was observed in the absence of SA using the PtNF/ERGO/Pt microelectrode (curve e). In the DPV curve (Fig. 2B), similar trends as that of $\mathrm{CV}$ curves were observed for the different modified electrodes. An oxidation peak with much higher peak height also appeared at the similar potential for the PtNF/ERGO/Pt microelectrode in the presence of SA (Fig. 2B, curve e).

\subsection{Optimization of conditions for detection in solution}

The concentration of $\mathrm{GO}$ and $\mathrm{H}_{2} \mathrm{PtCl}_{6} \cdot 6 \mathrm{H}_{2} \mathrm{O}$ and the scan cycles of electrodeposition were optimized as they are the key factors in the electrodeposition process. As shown in Fig. 3A, with the increase of the concentration of GO from 0 to $1 \mathrm{mg} \mathrm{ml}^{-1}$, the peak current of SA increases. While further increasing the concentration of GO, the peak current of SA decreases. So the optimal concentration of $\mathrm{GO}$ is $1 \mathrm{mg} \mathrm{m}{ }^{-1}$. The optimal conditions for $\mathrm{H}_{2} \mathrm{PtCl}_{6} \cdot 6 \mathrm{H}_{2} \mathrm{O}$ and scan cycles are $10 \mathrm{mM}$ and 45 cycles respectively as can be seen from Fig. $3 \mathrm{~B}$ and C.

\subsection{Performance of PtNF/ERGO/Pt microsensor}

The modified electrode was used to detect different concentrations of SA standard solution using DPV method. As shown in Fig. $4 \mathrm{~A}$, with the increasing concentrations of $\mathrm{SA}$, the peak current of SA increases correspondingly. There is a decent linear relation between the peak currents and the logarithm value of the concentrations of SA from $100 \mathrm{pM}$ to $1 \mu \mathrm{M}$ (Fig. 4B). And the equation is $I_{\text {peak }}(\mu \mathrm{A})=0.45 \mathrm{LN} C_{\mathrm{SA}}(\mathrm{nM})+1.05$, the correlation coefficient is 0.9807 . The detection limit of this sensor is $48.11 \mathrm{pM}(\mathrm{S} / \mathrm{N}=3.0)$. The sensing performance of the $\mathrm{PtNF} / \mathrm{ERGO} / \mathrm{Pt}$ microsensor for $\mathrm{SA}$ is compared with the previous reports. As shown in Table S1, $\dagger$ the developed sensor in this work is superior to most electrochemical sensors existed for SA. . $^{211,29-33}$

Selectivity is an important parameter to evaluate the performance of the sensor. Therefore, the possible interference in the seedling stem sap was examined by the proposed sensor under the same condition. As shown in Fig. 5, an oxidation peak around $1.15 \mathrm{~V}$ is observed for SA. Besides, a weak peak near $+0.8 \mathrm{~V}$ is observed for indole-3-acetic acid with the same concentration, which is consistent with previous report. ${ }^{11}$ However, it does not interfere with the detection of SA. Other components including malic acid, citric acid, succinic acid, abscisic acid and methyl jasmonate don't show any responses in the DPV curve. This result suggests the high selectivity of the proposed microsensor. The RSD was $15 \%$ for 8 different PtNF/
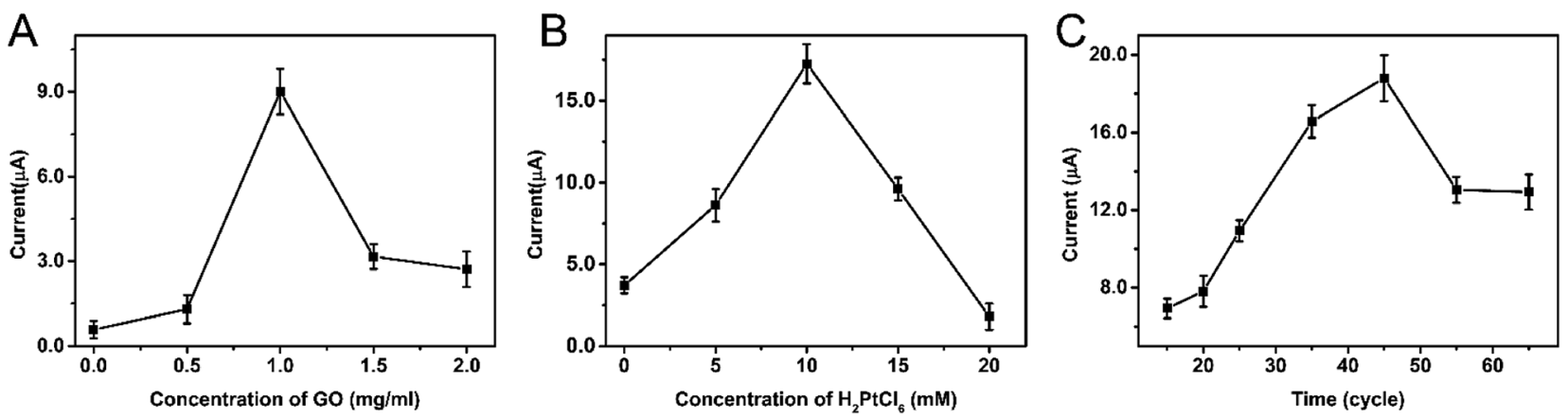

Fig. 3 Optimization of the modification process with different concentrations of $\mathrm{GO}(\mathrm{A}), \mathrm{H}_{2} \mathrm{PtCl}_{6}$ (B), and different scan cycles for deposition (C). 
A
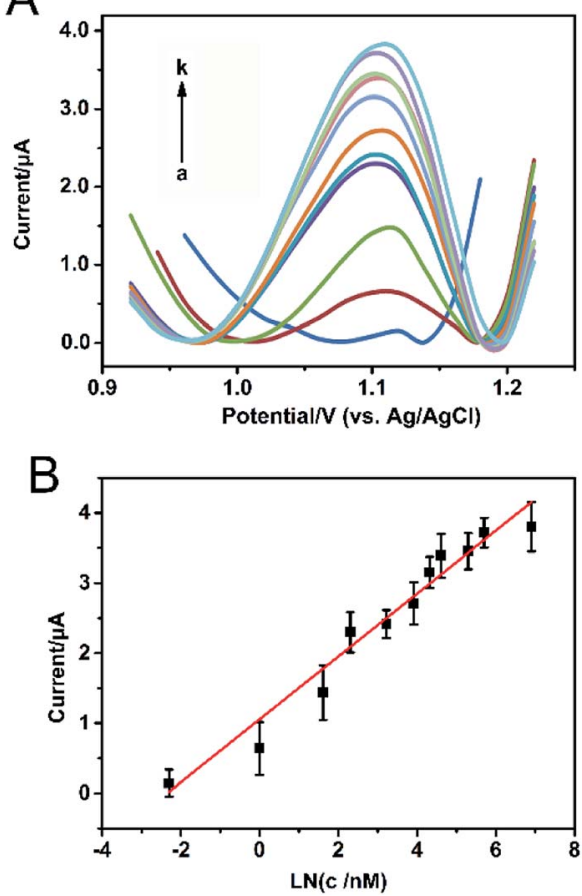

Fig. 4 (A) DPV curves of SA with concentrations of $0.1,1.0,5.0,10.0$, 25.0, 50.0, 75.0, 100.0, 200.0, 300.0, $1000 \mathrm{nM}$ (from a to $\mathrm{k}$ in turn) detected by the PtNF/ERGO/Pt electrode. (B) The calibration curve of the DPV peak current and the logarithm value of SA concentrations in PBS buffer. The average values and standard deviations were obtained based on five results.

ERGO/Pt microelectrodes in the presence of $500 \mu \mathrm{M}$ SA. There is still $86 \%$ sensing ability remained for the microsensor after storage at $4{ }^{\circ} \mathrm{C}$ for three weeks, indicating that this microsensor is highly stable.

In order to obtain a standard curve which is more suitable to be applied in the living plants, ${ }^{34}$ the DPV responses of modified electrode for SA was also detected in 10\% homogenate of sunflower stems diluted by 0.1 M PBS (Fig.S1 $\dagger$ ). As shown in Fig. 6, the linear equation is $I_{\text {peak }}^{\prime}(\mu \mathrm{A})=0.088 \mathrm{LNC}_{\mathrm{SA}}^{\prime}(\mathrm{nM})+0.993$ for SA from $10 \mathrm{pM}$ to $500 \mathrm{nM}$. The detection limit is $3.3 \mathrm{pM}$ (at $\mathrm{S} / \mathrm{N}$ of 3.0), and correlation coefficient is 0.9811 .

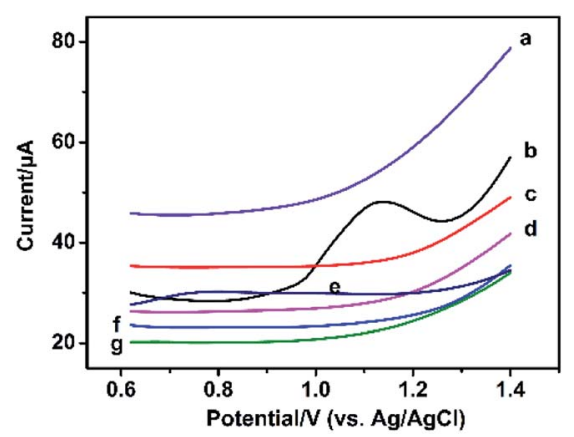

Fig. 5 DPV responses of $500 \mu \mathrm{M}$ methyl jasmonate (a), malic acid (c), succinic acid (d), indole-3-acetic acid (e), citric acid (f), abscisic acid (g), and salicylic acid (b) detected by the PtNF/ERGO/Pt electrode.

\subsection{In vivo measurements of $\mathrm{SA}$ in sunflower seedlings under salt stress}

Salt stress is one of the major abiotic environmental factors which adversely affect plant growth and development. Phytohormones play critical roles in regulating plant responses to salt stress. ${ }^{35}$ In this work, the modified PtNF/ERGO/Pt microelectrode was used to detect the SA levels in vivo in sunflower seedlings under different salt stress. Fig. 7A is the photograph for the in vivo detection of SA in sunflower seedlings. Fig. 7B shows the typical DPV results for the in vivo detection of SA in sunflower seedlings. The concentration of SA in the stem part of sunflowers is $256.1 \pm 0.32 \mathrm{nM}, 179.5 \pm 0.25 \mathrm{nM}$, and $158.6 \pm$ $0.15 \mathrm{nM}$ for $0 \mathrm{mM}, 50 \mathrm{mM}$ and $100 \mathrm{mM}$ of NaCl treatments $(10$ h), respectively, according to the standard curve in $10 \%$ homogenate of sunflower stems. This result suggests that with the increasing concentrations of salt, the SA levels decreases. This result is consistent with previous studies. ${ }^{31,36}$ In addition, the diameter of the microelectrode is only $0.5 \mathrm{~mm}$, only tiny holes were left after the detection. And the electrochemical test is so fast that there is no much influences were observed on the following growth of the plants.

In order to verify our result, UPLC-MS method was used to measure the SA levels in the extracted samples of the stem part of sunflower seedlings after different treatment of salt stress. The obtained result is shown in Fig. 7C, it could be found that the trend of SA levels is decreased with the increasing concentrations of salt, which is consistent with those obtained by the proposed electrochemical sensor.

The expression of SA related gene in sunflowers was also analyzed by RT-PCR method. The product of def is defensin (PR12 protein). It is a kind of antifungus, which can be impacted by $\mathrm{SA}^{20}$ HaAC1 encodes aldoketo reductase, which also is a marker of SA pathway in sunflowers. ${ }^{37}$ As shown in Fig. 7D, the expression of HaAC1 gene is down-regulated with the increasing concentrations of salt. The expression of def gene is slightly up-regulated for the salt-treated groups, but for the $50 \mathrm{mM}$ and $100 \mathrm{mM}$ of salt treatment, the expression change of def gene is not obvious. Similar trend of SA levels and the expression of def and HaAC1gene are also observed for sunflowers when it was attacked by Orobanche Cumana. ${ }^{\mathbf{2 0}}$

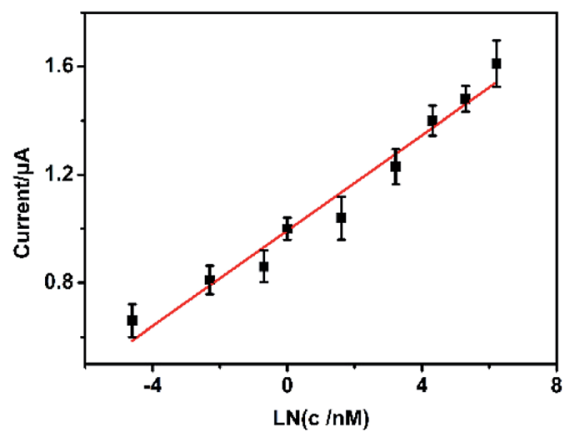

Fig. 6 The calibration curve of the DPV peak current and the logarithm of SA concentrations $(0.01,0.1,0.5,1.0,5.0,25.0,75.0,200.0$, $500.0 \mathrm{nM}$ in turn) in a $10 \%$ homogenate of sunflower seedling stems diluted by $0.01 \mathrm{M}$ PBS. 
A

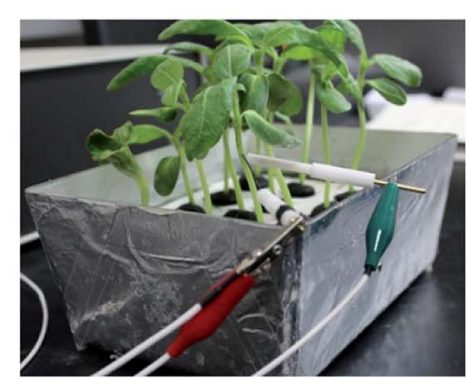

C

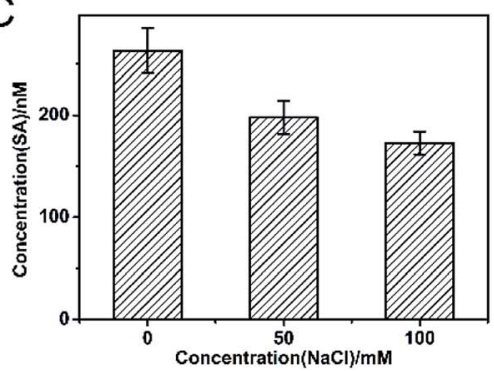

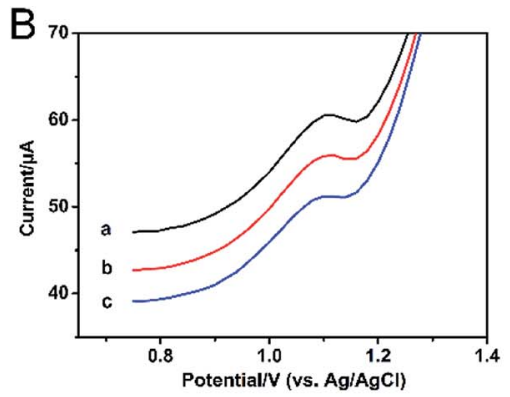

D

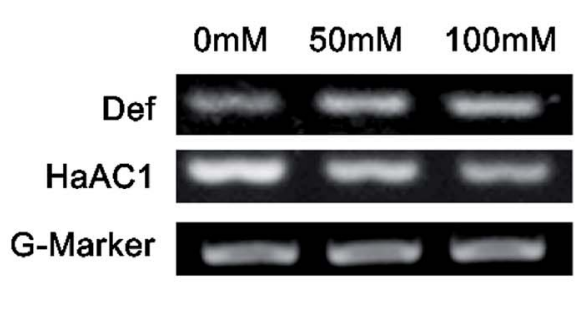

Fig. 7 (A) The real image of in vivo detection of SA in a sunflower seedling stem by the PtNF/ERGO/Pt electrode. (B) Typical DPV curves for the in vivo detection of SA by the PtNF/ERGO/Pt electrode under different salt stress: $0 \mathrm{mM}$ (a), $50 \mathrm{mM}$ (b) and $100 \mathrm{mM}$ (c). (C) UPLC-MS result of SA levels in the stem of sunflower seedlings. (D) Gene expression analysis of def, HaAC1gene relative to G-Marker in the stem of sunflower seedlings.

\section{Conclusions}

In summary, the PtNF/ERGO/Pt microelectrode was developed to detect SA in the stem of sunflower seedlings under different salt stress in vivo. Our result was consistent with previous report, and also confirmed by UPLC-MS and gene expression analysis. This is the first time for in vivo detection of SA in plants using microelectrode. The proposed SA microsensor is simple and practical, and showed high sensitivity and selectivity. It is foreseeable that our strategy could pave the way for the in vivo detection of phytohormone in plants.

\section{Conflicts of interest}

There are no conflicts to declare.

\section{Acknowledgements}

The authors are thankful for the fundings from Beijing Natural Science Foundation (2182022), Scientific and Technological Innovation Team of Beijing Academy of Agricultural and Forestry Sciences (JNKYT201604), and National Natural Science Foundation of China (Grant No. 61571443).

\section{Notes and references}

1 B. Lacombe and P. Achard, Curr. Opin. Plant Biol., 2016, 34, 1-8.

2 Z. Wang, F. Ai, Q. Xu, Q. Yang, J. H. Yu, W. H. Huang and Y. D. Zhao, Colloids Surf., B, 2010, 76, 370-374.

3 S. Hayat, A. Ahmad and M. N. Alyemeni, Salicylic acid: plant growth and development, Springer, Netherlands, 2013.
4 S. Croubels, M. An, K. Baert and P. D. Backer, Anal. Chim. Acta, 2005, 529, 179-187.

5 R. A. Budi Muljono, A. M. G. Looman, R. Verpoorte and J. J. C. Scheffer, Phytochem. Anal., 2015, 9, 35-38.

6 J. Sun, B. Wang, Z. Xue, Z. Li and X. Yang, Anal. Chem., 2016, 88, 1355-1361.

7 M. M. Sena, M. G. Trevisan and R. J. Poppi, Talanta, 2006, 68, 1707-1712.

8 P. J. Tseng, C. Y. Wang, T. Y. Huang, Y. Y. Chuang, S. F. Fu and Y. W. Lin, Anal. Methods, 2014, 6, 1759-1765.

9 W. Huang, L. Huang, G. Preston, M. Naylor, J. Carr, Y. Li, A. Singer, A. Whiteley and H. Wang, Plant J., 2010, 46, 1073-1083.

10 M. I. Gonzálezsánchez, P. T. Lee, R. H. Guy and R. G. Compton, Chem. Commun., 2015, 51, 16534-16536.

11 L. Sun, Q. Feng, Y. Yan, Z. Pan, X. Li, F. Song, H. Yang, J. Xu, N. Bao and H. Gu, Biosens. Bioelectron., 2014, 60, 154-160.

12 Y. Liu, G. Sun, C. Jiang, X. Zheng, L. Zheng and C. Li, Microchim. Acta, 2014, 181, 63-70.

13 Y. Ning, Y. Chen, Y. Shen, Y. Tang, J. Guo, F. Fang and S. Liu, J. Electrochem. Soc., 2013, 160, H715-H719.

14 Q. Xu, F. Wei, Z. Wang, Q. Yang, Y. D. Zhao and H. Chen, Sens. Actuators, B, 2009, 141, 599-603.

15 Q. Q. Ren, X. R. Huang, G. C. Liu, J. Ou-Yang, M. T. Li, H. Chen, Y. D. Zhao and W. Chen, Sens. Actuators, B, 2015, 220, 743-748.

16 Q. Xu, F. Wei, Z. Wang, Q. Yang, Y. D. Zhao and H. Chen, Phytochem. Anal., 2010, 21, 192-196.

17 A. S. Lima, K. R. Prieto, C. S. Santos, V. H. Paula, E. Y. Garciaochoa, A. Huertarobles, M. J. Beltrangarcia, M. P. Di and M. Bertotti, Biosens. Bioelectron., 2018, 99, 108-114. 
18 A. L. Liu, G. X. Zhong, J. Y. Chen, S. H. Weng, H. N. Huang, W. Chen, L. Q. Lin, Y. Lei, F. H. Fu and Z. L. Sun, Anal. Chim. Acta, 2013, 767, 50-58.

19 H. Gao, F. Xiao, B. C. Chi and H. Duan, ACS Appl. Mater. Interfaces, 2011, 3, 3049-3057.

20 X. Hu, D. L. Bidney, N. Yalpani, J. P. Duvick, O. Crasta, O. Folkerts and G. Lu, Plant Physiol., 2003, 133, 170-181.

21 C. Liu, K. Wang, S. Luo, Y. Tang and L. Chen, Small, 2011, 7, 1203-1206.

22 J. Yang, C. Tian, L. Wang and H. Fu, J. Mater. Chem., 2011, 21, 3384-3390.

23 M. Zhu, P. Chen and M. Liu, Langmuir, 2012, 28, 3385-3390.

24 C. V. Rao, A. L. M. Reddy, Y. Ishikawa and P. M. Ajayan, Carbon, 2011, 49, 931-936.

25 C. V. Rao, C. R. Cabrera and Y. Ishikawa, J. Phys. Chem. C, 2011, 115, 21963-21970.

26 Y. Hu, P. Wu, H. Zhang and C. Cai, Electrochim. Acta, 2012, 85, 314-321.

27 L. J. Cote, R. Cruzsilva and J. Huang, J. Am. Chem. Soc., 2009, 131, 11027-11032.
28 Y. Si and E. T. Samulski, Chem. Mater., 2008, 20, 6792-6797. 29 L. Lu, X. Zhu, X. Qiu, H. He, J. Xu and X. Wang, Int. J. Electrochem. Sci., 2014, 9, 8057-8066.

30 J. Park and C. Eun, Electrochim. Acta, 2016, 194, 346-356.

31 L. Sun, X. Liu, L. Gao, Y. Lu, Y. Li, Z. Pan, N. Bao and H. Gu, Anal. Lett., 2015, 48, 1578-1592.

32 Z. Wang, F. Wei, S. Y. Liu, Q. Xu, J. Y. Huang, X. Y. Dong, J. H. Yu, Q. Yang, Y. D. Zhao and H. Chen, Talanta, 2010, 80, 1277-1281.

33 M. H. A. Zavar, S. Heydari and G. H. Rounaghi, Arabian J. Sci. Eng., 2013, 38, 29-36.

34 W. Chen, Q. Yang, Q. Q. Ren, W. Wen, D. H. Jiang and Y. D. Zhao, Nanosci. Nanotechnol., 2013, 5, 694-698.

35 A. Molina and J. P. Donaire, New Phytol., 2010, 156, 409-415. 36 M. G. Javid, A. Sorooshzadeh, F. Moradi, S. A. M. M. Sanavy and I. Allahdadi, Aust. J. Crop Sci., 2011, 5, 726-734.

37 P. Letousey, A. De Zélicourt, D. S. C. Vieira, S. Thoiron, F. Monteau, P. Simier, P. Thalouarn and P. Delavault, Plant Pathol., 2007, 56, 536-546. 